\title{
Stage IIB Fallopian Tube Cancer AJCC v6 and $v 7$
}

National Cancer Institute

\section{Source}

National Cancer Institute. Stage /IB Fallopian Tube Cancer A/CC v6 and v7. NCI

Thesaurus. Code C6277.

Stage IIB includes: T2b, N0, M0. T2b: Extension to other pelvic structures. N0: No regional lymph node metastasis. M0: No distant metastasis. (AJCC 6th and 7th eds.) 